
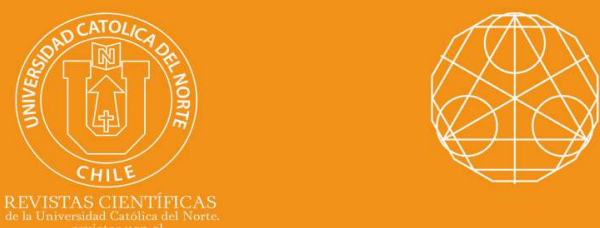

\title{
Applications of measure of noncompactness for the solvability of an infinite system of second order differential equations in some integrated sequence spaces
}

Rituparna Das ${ }^{1}$ orcid.org/0000-0003-0288-1326

Niraj Sapkota²®orcid.org/0000-0002-2176-9279

${ }^{1}$ Pandu College, Dept. of Mathematics, Guwahati, AS, India.

ri2p.das@gmail.com

${ }^{2}$ Sikkim Manipal University, Dept. of Mathematics, Gangtok, SK, India.

niraj.sapkota13@gmail.com.

Received: January 2020 | Accepted: November 2020

\section{Abstract:}

The aim of this paper is to study the infinite system of second order differential equations along with the given boundary conditions for its solvability in some integrated sequence spaces. The result is achieved with the analytical tool namely the measure of noncompactness along with the idea of MeirKeeler condensing operator and provides the realization of the sufficient conditions for the existence results in these Banach Sequence spaces. We also illustrate the results with examples.

Keywords: Sequence spaces; Measures of noncompactness; Infinite system of differential equations; Fixed point theory; Meir-Keeler condensing operators.

MSC (2020): 47H09, 47H10, 34A34.

\section{Cite this article as (IEEE citation style):}

R. Das and N. Sapkota, "Applications of measure of noncompactness for the solvability of an infinite system of second order differential equations in some integrated sequence spaces", Proyecciones (Antofagasta, On line), vol. 40, no. 2, pp. 573-592, 2021, doi: 10.22199/issn.0717-6279-2021-02-0032

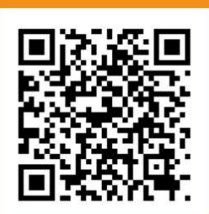

Article copyright: (C) 2021 Rituparna Das and Niraj Sapkota. This is an open access article distributed under the terms of the Creative Commons License, which permits unrestricted use and distribution provided the original author and source are credited. 


\section{Introduction}

The study of dynamical systems involves various types of calculus equations. There are several existence theorems for solutions in literature predating to 18th century. But infinite systems of equations (Differential, Integral, Integro-Differential etc.) is rather a new fascination. The theory of measure of noncompactness (MNC) has it's wide use as a tool in non- linear functional analysis. It finds its use in investigating the theories of differential equations and many other types of calculus equations and operator theories as well as fixed point theory. Over the years Kuratowski [1], Goldenštein and Markus [3], Istrăţescu [6] introduced the idea of different types of measure of noncompactness. In 1980, Banás and Goebel [7] gavethe axiomatic definition for MNC. Since many years MNC has been an efficient tool of analysis in various branches of mathematics. Existence andsolvability conditions for infinite system of differential equations of variousorders after its introduction by Persidskii $[8,9,10]$ led to several works in the line of MNC. Over the years, several authors have applied the concept of MNC in various branches of mathematics. Banaś and Lecko [15] proved the solvability of infinite systems of differential equations in Banach sequence spaces. Mursaleen and Mohiuddine [19] proved existence theoremsfor the infinite system of differential equations in the sequence space $\ell_{p}$. Srivastava et al. [26] studied the existence of solutions of infinite systemsof differential equations of general order with boundary conditions in thespaces $c_{0}$ and $\ell_{1}$ via the MNC. Mursaleen and Rizvi [25] proved existence of infinite systems of second order differential equations in the sequencespaces $c_{0}$ and $\ell_{1}$ by Meir-Keeler condensing operators. Alotaibi et al. [23] discussed the solvability of infinite system of linear equations in sequencespaces. Aghajani et al. [21] gave a generalization of Darbo's fixed pointtheorem with application to the solvability of systems of integral equations. Agarwal et al. [17] discussed the application of MNC to the existence ofsolutions for fractional differential equations. Mursaleen and Noman [18] discussed compactness of some operators by the Hausdorff MNC. Apart from these, many more applications of MNC's can be obtained in [11], [13],[14], [27], [28], [29].

The aim of this paper is to study the existence of solutions of the second order differential equation

$$
\left(D^{2}+1\right) u_{i}=f_{i}\left(t, u_{1}, u_{2}, u_{3}, \ldots\right) ; i=1,2,3, \cdots
$$

in the sequence spaces $\int \ell_{1}$ and $\int b v$ where, $u_{i}(0)=u_{i}(T)=0, \quad t \in$ 
$[0, T], \quad 0<T<\pi$ and $D^{2} \equiv \frac{d^{2}}{d t^{2}}$.

Here, notation $\int \ell_{1}$ and $\int b v$ represents integrated sequence spaces of well known sequence spaces $\ell_{1}$ and $b v$ respectively. The concept of integrated sequence space was initiated by Goes and Goes [5]. If $E$ is any sequence space, then $\int E=\left\{x=\left(x_{k}\right):\left\{k x_{k}\right\} \in E\right\}$ is defined as the integrated space of $E$. And hence by definition,

$$
\begin{aligned}
& \int \ell_{1}=\left\{x=\left(x_{k}\right): \sum_{k}\left|k x_{k}\right|<\infty\right\} \\
& \int b v=\left\{x=\left(x_{k}\right): \sum_{k}\left|k x_{k}-(k-1) x_{k-1}\right|<\infty\right\} .
\end{aligned}
$$

\section{Preliminaries and Background}

Let $(X,\|\|$.$) be a Banach space over \mathbf{R}$. Let $M_{X}$ be the family of all nonempty and bounded subsets of $X$ and let $M_{X}^{C}$ be the subfamily of $M_{X}$ consisting of all closed sets. Further, let $N_{X}$ be the family of all non-empty and relatively compact subsets of $X$. If $E \subseteq X$, then by $\bar{E}$ and $\operatorname{Conv}(E)$, we denote the closure of $E$ and convex closure of $E$ respectively.

The following definition of measure of noncompactness is due to Banaś and Goebel [7].

Definition 2.1. Let $X$ be a Banach space. A function $\mu: M_{X} \rightarrow[0,+\infty)$ is said to be a measure of noncompactness in $X$ if it satisfies the following axioms:

1. The family ker $\mu=\left\{A \in M_{X}: \mu(A)=0\right\}$ is nonempty and ker $\mu \subset$ $N_{X}$.

2. $A_{1} \subset A_{2} \Rightarrow \mu\left(A_{1}\right) \leq \mu\left(A_{2}\right)$.

3. $\mu(\bar{A})=\mu(A)$.

4. $\mu(\operatorname{Conv}(A))=\mu(A)$.

5. $\mu(\lambda A+(1-\lambda) B) \leq \lambda \mu(A)+(1-\lambda) \mu(B)$ for all $\lambda \in(0,1)$.

6. If $\left(F_{n}\right)$ is a decreasing sequence in $M_{X}^{C}$ and $\lim _{n \rightarrow \infty} \mu\left(F_{n}\right)=0$, then $\bigcap_{n=1}^{\infty} F_{n} \neq \emptyset$. 
The following definitions and results will be used in order to establish the results of this paper.

Definition 2.2. ( Akhmerov et. al. [12]) Let $\mu_{1}$ and $\mu_{2}$ be two arbitrary measures of noncompactness on the Banach spaces $E_{1}$ and $E_{2}$ respectively. $A\left(\mu_{1}, \mu_{2}\right)$-condensing operator is defined as an operator $F: E_{1} \rightarrow E_{2}$ such that

1. $F$ is continuous,

2. for every set $A$ in $E_{1}$ which is bounded as well as noncompact, we have $\mu_{2}(F(A))<\mu_{1}(A)$.

It is to be noted that a $\left(\mu_{1}, \mu_{2}\right)$-condensing operator is called a $\mu$-condensing operator if $\mu_{1}=\mu_{2}=\mu$ and is defined on the same Banach space.

Theorem 2.1. (Darbo [2]) Let $E$ be a Banach space and $A \in M_{E}^{C}$. A continuous mapping $F: A \rightarrow A$ has a fixed point in $A$ if

1. $A$ is convex,

2. $\mu(F(A)) \leq k \mu(A)$ for some $k \in[0,1)$ and for any arbitrary measure of noncompactness $\mu$ in $E$.

Definition 2.3. (Meir and Keeler [4]) Let $(E, d)$ be a metric space. A Meeir-Keeler contraction on $E$ is defined as a mapping $F$ on $E$ such that for any $\epsilon>0$, there exists $\delta>0$ with $d(F x, F y)<\epsilon$ whenever $\epsilon \leq d(x, y)<$ $\epsilon+\delta$ for all $x, y \in E$.

Theorem 2.2. (Meir and Keeler [4]) A Meir-Keeler contraction F on a metric space $(E, d)$ has a unique fixed point if $E$ is complete.

Definition 2.4. (Aghajani et. al. [22]) Let $\mu$ be an arbitrary measure of noncompactness on a Banach space $E$ and let $C$ be a nonempty subset of $E$. A Meir-Keeler condensing operator on $C$ is defined as an operator $F: C \rightarrow C$ such that for any $\epsilon>0$, there exists $\delta>0$ with $\mu(F(B))<\epsilon$ whenever $\epsilon \leq \mu(B)<\epsilon+\delta$ for any bounded subset $B$ of $C$.

Theorem 2.3. (Aghajani et. al. [22]) Let $E$ be a Banach space and $A \in M_{E}^{C}$. Let $\mu$ be an arbitrary measure of noncompactness on E. A continuous mapping $F: A \rightarrow A$ has at least one fixed point and the set of all fixed points of $F$ in $A$ is compact if 
1. $A$ is convex,

2. $F$ is a Meir-Keeler condensing operator.

Now, we shall recall some definitions related to BK-Space and AKSpace.

Definition 2.5. (Banaś and Mursaleen [20]) A metric space $(X, d)$, where $X$ is a linear space over $\mathbf{K}(=\mathbf{R}$ or $\mathbf{C})$, is said to be a linear metric space if and only if

1. $d(x+z, y+z)=d(x, y)$, for all $x, y, z \in X$.

2. the vector addition $(x, y) \rightarrow x+y$ is a continuous function from $X \times X$ into $X$.

3. the scalar multiplication map $(\lambda, x) \rightarrow \lambda x$ is a continuous function from $\mathbf{K} \times X$ into $X$.

Definition 2.6. (Banaś and Mursaleen [20]) A complete linear metric space is called a Fréchet space.

Definition 2.7. (Banaś and Mursaleen [20]) A linear topological space (or topological vector space) $X$ is a linear space (vector space) over $\mathbf{K}(=$ $\mathbf{R}$ or $\mathbf{C})$ which is endowed with a topology such that vector addition $X \times$ $X \rightarrow X$ and scalar multiplication $\mathbf{K} \times X \rightarrow X$ are continuous functions.

Throughout $w$ denotes the space of all real or complex valued sequences. By $e^{(n)}=\left\{e_{1}^{(n)}, e_{2}^{(n)}, e_{3}^{(n)}, \cdots\right\},(n=1,2, \cdots)$, we denote the sequence such that $e_{n}^{(n)}=1$ and $e_{k}^{(n)}=0$ for $k=n$. For any sequence $x=\left(x_{k}\right)$, let $x^{[n]}=\sum_{k=1}^{n} x_{k} e^{(k)}$ be its $n$-section.

\section{Definition 2.8. (Banaś and Mursaleen [20])}

1. A sequence space $X$ with a linear topology is said to be a $K$-space if each of the maps $p_{n}: X \rightarrow \mathbf{C}$ defined by $p_{n}(x)=x_{n}$ is continuous for each $n \in \mathbf{N}$.

2. A K-space is said to be an FK-space if $X$ is a complete linear metric space, that is, $X$ is an FK-space if $X$ is Fréchet space with continuous coordinates. 
3. A normed FK-space is called a BK-space, that is, a BK-space is a Banach sequence space with continuous coordinates.

4. A sequence $\left(b^{(k)}\right)_{k=1}^{\infty}$ in a linear metric space $X$ is called a Schauder basis for $X$ if for every $x \in X$, there exists a unique sequence $\left(\lambda_{n}\right)_{n=1}^{\infty}$ of scalars such that $x=\sum_{n=1}^{\infty} \lambda_{n} b^{(n)}$.

5. An FK-space $X$ is said to have $A K$ if every sequence $x=\left(x_{n}\right) \in X$ has a unique representation $x=\sum_{k=1}^{\infty} x_{k} e^{(k)}$, that is, $x=\lim _{n \rightarrow \infty} x^{[n]}$. An FK-space with AK property is also called an AK-space.

\section{Hausdorff Measure of Noncompactness on Banach Spaces}

Definition 3.1. Let $Q$ a bounded subset of a metric space $(X, d)$. Then the Hausdorff measure of noncompactness of $Q$, denoted by $\mathcal{X}(Q)$, is defined as

$\left(3.1 \mathcal{X}(Q)=\inf \left\{\epsilon>0: Q \subset \bigcup_{i=1}^{n} B\left(x_{i}, r_{i}\right), x_{i} \in X, r_{i}<\epsilon, 1 \leq i \leq n, n \in \mathbf{N}\right\}\right.$

where, $B\left(x_{i}, r_{i}\right)$ is an open ball in $X$.

Theorem 3.1. (Banaś and Mursaleen [20]) Let $X$ be a BK space with $A K$ and monotone norm, $Q \in M_{X}, P_{n}: X \rightarrow X,(n \in \mathbf{N})$ be the operator (projection) defined by $P_{n}\left(x_{1}, x_{2}, \cdots\right)=x^{[n]}=\left(x_{1}, x_{2}, \cdots, x_{n}, 0,0, \cdots\right)$ for all $x=\left(x_{1}, x_{2}, \cdots\right) \in X$. Then

$$
\mathcal{X}(Q)=\lim _{n \rightarrow \infty}\left(\sup _{x \in Q}\left\|\left(I-P_{n}\right)(x)\right\|\right) .
$$

Kirişci [24] proved that the integrated sequence spaces $\int \ell_{1}$ and $\int b v$ are $B K$-spaces with $A K$-property with respect to the norms

$$
\|x\|_{\int \ell_{1}}=\sum_{k}\left|k x_{k}\right| \text { and }\|x\|_{\int b v}=\sum_{k}\left|k x_{k}-(k-1) x_{k-1}\right|
$$

respectively, where the norms are also monotone.

In view of the Theorem (3.1), in the Banach Sequence Space $\left(\int \ell_{1},\|\cdot\| \int \ell_{1}\right)$, the Hausdorff measure of noncompactness $\mathcal{X}$ can be formulated as

$$
\mathcal{X}(B)=\lim _{n \rightarrow \infty}\left\{\sup _{u \in B}\left[\sum_{k \geq n}\left|k u_{k}\right|\right]\right\}
$$


where, $u(t)=\left(u_{i}(t)\right)_{i=1}^{\infty} \in \int \ell_{1}$ for each $t \in[0, T]$ and $B \in M_{\int \ell_{1}}$.

In the Banach Sequence Space $\left(\int b v,\|\cdot\|_{\int b v}\right)$, the Hausdorff measure of noncompactness $\mathcal{X}$ can be formulated as

$$
\mathcal{X}(B)=\lim _{n \rightarrow \infty}\left\{\sup _{u \in B}\left[\sum_{k \geq n}\left|k u_{k}-(k-1) u_{k-1}\right|\right]\right\}
$$

where, $u(t)=\left(u_{i}(t)\right)_{i=1}^{\infty} \in \int b v$ for each $t \in[0, T]$ and $B \in M_{\int b v}$.

In this study, we consider the following infinite system of second order differential equations

$$
\left(D^{2}+1\right) u_{i}=f_{i}\left(t, u_{1}, u_{2}, u_{3}, \ldots\right) ;{ }^{\prime} i=1,2,3, \cdots
$$

where, $u_{i}(0)=u_{i}(T)=0, \quad t \in[0, T], \quad 0<T<\pi$ and $D^{2} \equiv \frac{d^{2}}{d t^{2}}$.

Let $C([0, T], \mathbf{R})$ denotes the space of all real valued continuous functions over $[0, T]$ and $C^{2}([0, T], \mathbf{R})$ denotes the set of all functions with the second continuous derivative on $[0, T]$. A function $u \in C^{2}([0, T], \mathbf{R})$ is a solution of (3.4) if and only if $u \in C([0, T], \mathbf{R})$ is a solution of the infinite system of integral equation

$$
u_{i}(t)=\int_{0}^{T} G(t, s) f_{i}(s, u(s)) d s, \text { for } t \in[0, T]
$$

where, $f_{i}(t, u) \in C([0, T], \mathbf{R}), i=1,2,3, \ldots$ The Green's function associated with the system (3.4) is given by (see Duffy [16])

$$
G(t, s)= \begin{cases}\frac{\sin (s-T) \sin t}{\sin T}, & 0 \leq t \leq s \leq T \\ \frac{\sin (t-T)}{\sin T}, & 0 \leq s \leq t \leq T\end{cases}
$$

From (3.5) and (3.6),

$$
u_{i}(t)=\int_{0}^{t} \frac{\sin s \sin (t-T)}{\sin T} f_{i}(s, u(s)) d s+\int_{t}^{T} \frac{\sin (s-T) \sin t}{\sin T} f_{i}(s, u(s)) d s
$$

Now,

$\frac{d}{d t} u_{i}(t)=\int_{0}^{t} \frac{\sin s \cos (t-T)}{\sin T} f_{i}(s, u(s)) d s+\int_{t}^{T} \frac{\sin (s-T) \cos t}{\sin T} f_{i}(s, u(s)) d s$

Again differentiating, we get 


$$
\begin{aligned}
\frac{d^{2}}{d t^{2}} u_{i}(t) & =\frac{\sin t \cos (t-T)}{\sin T} f_{i}(t, u(t))-\int_{0}^{t} \frac{\sin s \sin (t-T)}{\sin T} f_{i}(s, u(s)) d s \\
& -\frac{\sin (t-T) \cos t}{\sin T} f_{i}(t, u(t))-\int_{t}^{T} \frac{\sin (s-T) \sin t}{\sin T} f_{i}(s, u(s)) d s \\
& =-u_{i}(t)+f_{i}(t, u(t))
\end{aligned}
$$

In this paper, we establish the solvability of the system (3.4) in Banach sequence spaces $\int \ell_{1}$ and $\int b v$.

\section{Solvability of the system of equations (3.4) in the sequence space $\int \ell_{1}$}

Let us assume that

(H1) The functions $f_{i}$ are defined on the set $[0, T] \times \mathbf{R}^{\infty}$ and take real values. The operator $f$ defined on the space $[0, T] \times \int \ell_{1}$ as

$$
(t, u) \mapsto(f u)(t)=\left(f_{1}(t, u), f_{2}(t, u), f_{3}(t, u), \ldots\right)
$$

is such that the class of all functions $((f u)(t))_{t \in[0, T]}$ is equicontinuous at every point of the space $\int \ell_{1}$.

(H2) The following inequality holds:

$$
\left|f_{n}\left(t, u_{1}, u_{2}, u_{3}, \ldots\right)\right| \leq \frac{g_{n}(t)}{n}+h_{n}(t)\left|u_{n}(t)\right|,
$$

where $g_{n}(t)$ and $h_{n}(t)$ are real functions defined and continuous on $I$, such that $\sum_{k \geq 1} g_{k}(t)$ converges uniformly on $[0, T]$ and the sequence $\left(h_{n}(t)\right)$ is equibounded on $[0, T]$.

We also assume that:

$$
\begin{aligned}
g(t) & =\sum_{k \geq 1} g_{k}(t), \text { for each } t \in[0, T] \\
G & =\sup \{g(t): t \in[0, T]\} \\
H & =\sup \left\{h_{n}(t): n \in \mathbf{N}, t \in[0, T]\right\}
\end{aligned}
$$

Theorem 4.1. Under the hypotheses (H1)-(H2), the infinite system of differential equations (3.4) has at least one solution $u(t)=\left(u_{i}(t)\right)$ whenever $\frac{H T}{\sin T}<1$ such that $u(t) \in \int \ell_{1}$, for all $t \in[0, T]$. 
Proof. From the relation (3.6) and the hypothesis (H2), we have for an arbitrary $t \in[0, T]$

$$
\begin{aligned}
\|u(t)\|_{\int \ell_{1}} & =\sum_{i=1}^{\infty}\left|i u_{i}(t)\right| \\
& =\sum_{i=1}^{\infty}\left|i \int_{0}^{T} G(t, s) f_{i}(s, u(s)) d s\right| \\
& \leq \sum_{i=1}^{\infty} i \int_{0}^{T}|G(t, s)|\left|f_{i}(s, u(s))\right| d s \\
& \leq \sum_{i=1}^{\infty} i \int_{0}^{T}|G(t, s)|\left(\frac{g_{i}(s)}{i}+h_{i}(s)\left|u_{i}(s)\right|\right) d s \\
& =\sum_{i=1}^{\infty} \int_{0}^{T}|G(t, s)| g_{i}(s) d s+\sum_{i=1}^{\infty} \int_{0}^{T} i|G(t, s)| h_{i}(s)\left|u_{i}(s)\right| d s \\
& \leq \int_{0}^{T} \sum_{i=1}^{\infty}|G(t, s)| g_{i}(s) d s+H \sum_{i=1}^{\infty} \int_{0}^{T}\left|i u_{i}(s) \| G(t, s)\right| d s \\
& \leq \frac{G T}{\sin T}+\frac{H T}{\sin T}\|u\| \int \ell_{1}
\end{aligned}
$$

Hence, we get

$$
\|u\|_{\int \ell_{1}} \leq \frac{G T}{\sin T-H T}=r
$$

Let $u^{0}(t)=\left(u_{i}^{0}(t)\right)$ where $u_{i}^{0}(t)=0, \forall t \in[0, T]$. Let $\bar{B}=\bar{B}\left(u^{0}, r_{1}\right)$, the closed ball centered at $u^{0}$ and radius $r_{1} \leq r$. Then $\bar{B}$ is a nonempty, bounded, closed and convex subset of $\int \ell_{1}$.

Let us consider the operator $\mathcal{F}=\left(\mathcal{F}_{i}\right)$ on $C([0, T], \bar{B})$, defined as follows: For $t \in[0, T]$,

$$
(\mathcal{F} u)(t)=\left\{\left(\mathcal{F}_{i} u\right)(t)\right\}=\left\{\int_{0}^{T} G(t, s) f_{i}(s, u(s)) d s\right\},
$$

where $u(t)=\left(u_{i}(t)\right) \in \bar{B}$ and $u_{i}(t) \in C([0, T], \mathbf{R})$.

Since, $\left(f_{i}(t, u(t))\right) \in \int \ell_{1}$ for each $t \in[0, T]$, so we have

$$
\sum_{i=1}^{\infty}\left|i\left(\mathcal{F}_{i} u\right)(t)\right|=\sum_{i=1}^{\infty}\left|\int_{0}^{T} i G(t, s) f_{i}(s, u(s))\right| d s
$$




$$
\begin{aligned}
& \leq \sum_{i=0}^{\infty} \int_{0}^{T} i|G(t, s)|\left(\frac{g_{i}(s)}{i}+h_{i}(s)\left|u_{i}(s)\right|\right) d s \\
& \leq \frac{G T}{\sin T}+\frac{H T}{\sin T}\|u\|_{\int_{\ell_{1}}}<\infty
\end{aligned}
$$

Therefore, $(\mathcal{F} u)(t)=\left\{\left(\mathcal{F}_{i} u\right)(t)\right\} \in \int \ell_{1}$ for each $t \in[0, T]$.

Also, we have

$$
\begin{array}{rlrl}
\left(\mathcal{F}_{i}\right)(0) & =\int_{0}^{T} G(0, s) f_{i}(s, u(s)) d s\left(\mathcal{F}_{i}\right)(T) & =\int_{0}^{T} G(T, s) f_{i}(s, u(s)) d s \\
& =\int_{0}^{T} 0 . f_{i}(s, u(s)) d s=0 & & =\int_{0}^{T} 0 . f_{i}(s, u(s)) d s=0
\end{array}
$$

Therefore, each $\left(\mathcal{F}_{i} u\right)(t)$ satisfies boundary conditions given in (3.4).

Since $\left\|(\mathcal{F} u)(t)-u^{0}(t)\right\|_{\int_{\ell_{1}}}=\|(\mathcal{F} u(t))\|_{\int \ell_{1}} \leq r$, therefore, it follows that $\mathcal{F}$ is a self mapping on $\bar{B}$.

Also, by the assumption (H1), it is clear that the operator $\mathcal{F}$ is continuous on $C([0, T], \bar{B})$. We now show that $\mathcal{F}$ is a Meir-Keeler condensing operator for which for any given $\epsilon>0$, we need to find $\delta>0$ such that $\mathcal{X}(\mathcal{F} \bar{B})<\epsilon$ whenever $\epsilon \leq \mathcal{X}(\bar{B})<\epsilon+\delta$. Using equation (3.2), we have

$$
\begin{aligned}
\mathcal{X}(\mathcal{F} \bar{B}) & =\lim _{n \rightarrow \infty}\left(\sup _{u(t) \in \bar{B}}\left[\sum_{k \geq n}\left|k \int_{0}^{T} G(t, s) f_{k}(s, u(s)) d s\right|\right]\right) \\
& \leq \lim _{n \rightarrow \infty}\left(\sup _{u(t) \in \bar{B}}\left[\sum_{k \geq n}|k| \int_{0}^{T}\left|G(t, s) f_{k}(s, u(s)) d s\right|\right]\right) \\
& \leq \lim _{n \rightarrow \infty}\left(\sup _{u(t) \in \bar{B}}\left[\sum_{k \geq n} k \int_{0}^{T}|G(t, s)|\left(\frac{g_{k}(s)}{k}+h_{k}(s)\left|u_{k}(s)\right|\right) d s\right]\right) \\
& =\lim _{n \rightarrow \infty}\left(\sup _{u(t) \in \bar{B}}\left[\sum_{k \geq n} \int_{0}^{T}|G(t, s)| g_{k}(s) d s+\sum_{k \geq n} k \int_{0}^{T}|G(t, s)| h_{k}(s)\left|u_{k}(s)\right| d s\right]\right) \\
& \leq \lim _{n \rightarrow \infty}\left(\sup _{u(t) \in \bar{B}}\left[\int_{0}^{T}|G(t, s)| \sum_{k \geq n} g_{k}(s) d s+H \sum_{k \geq n} k\left|u_{k}\right| \int_{0}^{T}|G(t, s)| d s\right]\right) \\
< & \lim _{n \rightarrow \infty} \frac{T}{\sin T} \sum_{k \geq n} g_{k}(s)+\frac{H T}{\sin T} \lim _{n \rightarrow \infty}\left(\sup _{u(t) \in B}\left[\sum_{k \geq n} k\left|u_{k}\right|\right]\right) \\
& =\frac{H T}{\sin T} \mathcal{X}(\bar{B})
\end{aligned}
$$


Thus, we get $\mathcal{X}(\mathcal{F} \bar{B})<\mathcal{X}(\bar{B}) \frac{H T}{\sin T}<\epsilon \Longrightarrow \mathcal{X}(\bar{B})<\frac{\epsilon \sin T}{H T}$.

Taking $\delta=\frac{\sin T-H T}{H T} \epsilon$, we get $\epsilon \leq \mathcal{X}(\bar{B})<\epsilon+\delta$. This shows that $\mathcal{F}$ is a Meir-Keeler condensing operator defined on the set $\bar{B} \subset \int \ell_{1}$ and so, it satisfies all conditions of Theorem (2.3). Therefore $\mathcal{F}$ has fixed point in $\bar{B}$, which is a solution of the system of equations (3.4).

Example 4.1. Let us consider the system of differential equations

$$
\text { (4.1) } \frac{d^{2} u_{n}(t)}{d t^{2}}+u_{n}(t)=\frac{\sqrt[n]{t}}{n 3^{n-1}}+\sum_{m=n}^{\infty} \frac{t \cos t u_{m}(t)}{m^{3}}, \quad n \in \mathbf{N}, t \in[0, T]
$$

where, $0<T<\pi$, and $u_{i}(0)=u_{i}(T)=0$, for each $i$.

Clearly, the functions $\frac{\sqrt[n]{t}}{n 3^{n-1}}$ and $\sum_{m=n}^{\infty} \frac{t \cos t u_{m}(t)}{m^{3}}$ are continuous on $[0, T]$, for each $n \in \mathbf{N}$.

Here, $f_{n}(t, u(t))=\frac{\sqrt[n]{t}}{n 3^{n-1}}+\sum_{m=n}^{\infty} \frac{t \cos t u_{m}(t)}{m^{3}}, n \in \mathbf{N}, t \in[0, T]$.

Now,

$$
\begin{aligned}
\sum_{k=1}^{\infty}\left|k f_{k}(t, u(t))\right| & =\sum_{k=1}^{\infty}\left|\frac{\sqrt[k]{t}}{3^{k-1}}+k \sum_{m=k}^{\infty} \frac{t \cos t u_{m}(t)}{m^{3}}\right| \\
& \leq \sum_{k=1}^{\infty} \frac{\sqrt[k]{t}}{3^{k-1}}+\sum_{k=1}^{\infty} k \sum_{m=k}^{\infty}\left|\frac{t \cos t u_{m}(t)}{m^{3}}\right| \\
& \leq \frac{3 T}{2}+\sum_{k=1}^{\infty} k \sum_{m=k}^{\infty} \frac{t}{m^{4}}\left|m u_{m}(t)\right| \\
& \leq \frac{3 T}{2}+T \sum_{k=1}^{\infty} \frac{1}{2}\left(\frac{1}{k}+\frac{1}{k^{2}}\right)\left|u_{k}(t)\right| \\
& <\frac{3 T}{2}+T \sum_{k=1}^{\infty}\left|k u_{k}(t)\right| \\
& =\frac{3 T}{2}+T\|u(t)\| \int \ell_{1}<\infty
\end{aligned}
$$

Therefore, for each $t \in[0, T],\left(f_{i}(t, u(t))\right) \in \int \ell_{1}$.

We now show that the hypothesis (H1) is satisfied. 
Let $\epsilon>0$ be arbitrary and $u(t)=\left(u_{n}(t)\right) \in \int \ell_{1}$. Then taking $v(t)=$ $\left(v_{n}(t)\right) \in \int \ell_{1}$ with $\|u(t)-v(t)\|_{\int \ell_{1}} \leq \delta(\epsilon)=\frac{\epsilon}{T}$, we have

$$
\begin{aligned}
|f(t, u(t))-f(t, v(t))| & =\sum_{m=n}^{\infty} \frac{t\left(u_{m}(t)-v_{m}(t)\right)}{m^{3}} \\
& \leq \sum_{m=1}^{\infty} \frac{t m\left(u_{m}(t)-v_{m}(t)\right)}{m^{4}} \\
& \leq T\|u(t)-v(t)\| \int_{\ell_{1}} \\
& \leq T \delta<\epsilon,
\end{aligned}
$$

which implies continuity as assumed in (H1).

Now, we show that assumption (H2) is satisfied.

We have

$$
\begin{aligned}
\left|f_{n}(t, u(t))\right| & =\left|\frac{\sqrt[n]{t}}{n 3^{n-1}}+\sum_{m=n}^{\infty} \frac{t \cos t u_{m}(t)}{m^{3}}\right| \\
& \leq \frac{1}{n} \frac{\sqrt{t}}{3^{n-1}}+\sum_{m=n}^{\infty} \frac{t}{m^{3}}\left|u_{m}(t)\right| \\
& \leq \frac{1}{n} \frac{\sqrt{t}}{3^{n-1}}+\sum_{m=1}^{\infty} \frac{t}{m^{3}}\left|u_{m}(t)\right| \\
& \leq \frac{g_{n}(t)}{n}+h_{n}(t)\left|u_{n}(t)\right| .
\end{aligned}
$$

The function $g_{n}(t)=\frac{\sqrt{t}}{3^{n-1}}$ is continuous and $\sum_{n \geq 1} g_{n}(t)$ converges uniformly to $\frac{3 \sqrt{t}}{2}$; also $h_{n}(t)=t \zeta(3)$ is continuous and the sequence $\left(h_{n}(t)\right)$ is equibounded on $[0, T]$ by $H=T \zeta(3)$. Also $\frac{H T}{\sin T}<1$ is satisfied by taking $T=0.7$ (i.e. $0 \leq t \leq 0.7<\pi$ ), which gives $H \approx 0.84144$ and $G \approx 1.05$.

Thus, from Theorem (4.1), for a suitable value of $r$, the operator $\mathcal{F}$ as defined in Theorem (4.1) on $\bar{B}\left(u^{0}, r\right)$ has a fixed point $u(t)=\left(u_{i}(t)\right) \in \int \ell_{1}$, which is a solution of system of equations (4.1). 


\section{Solvability of the system of equations (3.4) in the sequence space $\int b v$}

Let us now consider the assumptions:

(H3) The functions $f_{i}$ are defined on the set $[0, T] \times \mathbf{R}^{\infty}$ and take real values. The operator $f$ defined on the space $[0, T] \times \int b v$ as

$$
(t, u) \mapsto(f u)(t)=\left(f_{1}(t, u), f_{2}(t, u), f_{3}(t, u), \ldots\right)
$$

is such that the class of all functions $((f u)(t))_{t \in[0, T]}$ is equicontinuous at every point of the space $\int b v$.

(H4) The following inequality holds:

$\left|n f_{n}\left(t, u_{1}, u_{2}, \ldots\right)-(n-1) f_{n-1}\left(t, u_{1}, u_{2}, \ldots\right)\right| \leq g_{n}(t)+h_{n}(t)\left|n u_{n}(t)-(n-1) u_{n-1}(t)\right|$

where $g_{n}(t)$ and $h_{n}(t)$ are real functions defined and continuous on $[0, T]$, such that $\sum_{k \geq 1} g_{k}(t)$ converges uniformly on $[0, T]$ and the sequence $\left(h_{n}(t)\right)$ is equibounded on $[0, T]$.

We also assume that:

$$
\begin{aligned}
g(t) & =\sum_{k \geq 1} g_{k}(t), \text { for each } t \in[0, T] \\
G & =\sup \{g(t): t \in[0, T]\} \\
H & =\sup \left\{h_{n}(t): n \in \mathbf{N}, t \in[0, T]\right\}
\end{aligned}
$$

Theorem 5.1. Under the hypotheses (H3)-(H4), the infinite system of differential equations (3.4) has at least one solution $u(t)=\left(u_{i}(t)\right)$ whenever $\frac{H T}{\sin T}<1$ such that $u(t) \in \int b v, \forall t \in[0, T]$.

Proof. Using the relation (3.3) and the hypothesis (H4), we have for an arbitrary $t \in[0, T]$,

$$
\begin{aligned}
\|u(t)\| \|_{j b v} & =\sum_{i=1}^{\infty}\left|i u_{i}(t)-(i-1) u_{i-1}(t)\right| \\
& =\sum_{i=1}^{\infty}\left|i \int_{0}^{T} G(t, s) f_{i}(s, u(s)) d s-(i-1) \int_{0}^{T} G(t, s) f_{i-1}(s, u(s)) d s\right|
\end{aligned}
$$




$$
\begin{aligned}
& \leq \sum_{i=1}^{\infty} \int_{0}^{T}|G(t, s)|\left|\left(i f_{i}(s, u(s)) d s-(i-1) f_{i-1}(s, u(s))\right)\right| d s \\
& \leq \sum_{i=1}^{\infty} \int_{0}^{T}|G(t, s)|\left(g_{i}(s)+h_{i}(s)\left|i u_{i}(s)-(i-1) u_{i-1}(s)\right|\right) d s \\
& =\sum_{i=1}^{\infty} \int_{0}^{T}|G(t, s)| g_{i}(s) d s+\sum_{i=1}^{\infty} \int_{0}^{T}|G(t, s)| h_{i}(s)\left|i u_{i}(s)-(i-1) u_{i-1}(s)\right| d s \\
& \leq \int_{0}^{T} \sum_{i=1}^{\infty}|G(t, s)| g_{i}(s) d s+H \sum_{i=1}^{\infty} \int_{0}^{T}\left|i u_{i}(s)-(i-1) u_{i-1}(s)\right| G(t, s) d s \\
& \leq \frac{G T}{\sin T}+\frac{H T}{\sin T}\|u \mid\|_{j} b v
\end{aligned}
$$

Hence, we get

$$
\|u\|_{\int b v} \leq \frac{G T}{\sin T-H T}=r
$$

Let $u^{0}(t)=\left(u_{i}^{0}(t)\right)$ where $u_{i}^{0}(t)=0, \forall t \in[0, T]$. Take $\bar{B}=\bar{B}\left(u^{0}, r_{1}\right)$, the closed ball centered at $u^{0}$ and radius $r_{1} \leq r$. Clearly, $\bar{B}$ is a nonempty, bounded, closed and convex subset of $\int b v$.

Let us consider the operator $\mathcal{F}=\left(\mathcal{F}_{i}\right)$ on $C([0, T], \bar{B})$, defined as follows:

$$
(\mathcal{F} u)(t)=\left\{\left(\mathcal{F}_{i} u\right)(t)\right\}=\left\{\int_{0}^{T} G(t, s) f_{i}(s, u(s)) d s\right\}, t \in[0, T]
$$

where $u(t)=\left(u_{i}(t)\right) \in \bar{B}$ and $u_{i}(t) \in C([0, T], \mathbf{R})$.

We first show that $(\mathcal{F} u)(t)=\left\{\left(\mathcal{F}_{i} u\right)(t)\right\} \in \int b v$ for each $t \in[0, T]$.

Since, $\left(f_{i}(t, u(t))\right) \in \int b v$ for each $t \in[0, T]$, so we have

$$
\begin{aligned}
\sum_{i=1}^{\infty} \mid i\left(\mathcal{F}_{i} u\right)(t) & -(i-1)\left(\mathcal{F}_{i-1} u\right)(t) \mid \\
& =\sum_{i=1}^{\infty}\left|i \int_{0}^{T} G(t, s) f_{i}(s, u(s)) d s-(i-1) \int_{0}^{T} G(t, s) f_{i-1}(s, u(s)) d s\right| \\
& =\sum_{i=1}^{\infty}\left|\int_{0}^{T} G(t, s)\left(i f_{i}(s, u(s))-(i-1) f_{i-1}(s, u(s))\right) d s\right| \\
& \leq \sum_{i=1}^{\infty} \int_{0}^{T}|G(t, s)|\left(g_{i}(s)+h_{i}(s)\left|i u_{i}(s)-(i-1) u_{i-1}(s)\right|\right) d s \\
& \leq \frac{G T}{\sin T}+\frac{H T}{\sin T}\|u\|_{\int b v}<\infty
\end{aligned}
$$


Therefore, $(\mathcal{F} u)(t)=\left\{\left(\mathcal{F}_{i} u\right)(t)\right\} \in \int b v$ for each $t \in[0, T]$.

Also, we have

$$
\begin{array}{rlrl}
\left(\mathcal{F}_{i}\right)(0) & =\int_{0}^{T} G(0, s) f_{i}(s, u(s)) d s\left(\mathcal{F}_{i}\right)(T) & =\int_{0}^{T} G(T, s) f_{i}(s, u(s)) d s \\
& =\int_{0}^{T} 0 . f_{i}(s, u(s)) d s=0 & & =\int_{0}^{T} 0 . f_{i}(s, u(s)) d s=0
\end{array}
$$

Therefore, each $\left(\mathcal{F}_{i} u\right)(t)$ satisfies boundary conditions given in the system (3.4).

Since $\left\|(\mathcal{F} u)(t)-u^{0}(t)\right\|_{\int b v}=\|(\mathcal{F} u(t))\|_{\int b v} \leq r$, thus $\mathcal{F}$ is a self mapping on $\bar{B}$. The operator $\mathcal{F}$ is continuous on $C([0, T], \bar{B})$ by the assumption (H3).

We now show that $\mathcal{F}$ is a Meir-Keeler condensing operator.

Let $\epsilon>0$. We need to find $\delta>0$ such that $\mathcal{X}(\mathcal{F} \bar{B})<\epsilon$ whenever $\epsilon \leq \mathcal{X}(\bar{B})<\epsilon+\delta$.

Using equation (3.3), we obtain

$$
\begin{aligned}
& \mathcal{X}(\mathcal{F} \bar{B}) \\
= & \lim _{n \rightarrow \infty}\left(\sup _{u(t) \in B}\left[\sum_{k \geq n}\left|k \int_{0}^{T} G(t, s) f_{k}(s, u(s)) d s-(k-1) \int_{0}^{T} G(t, s) f_{k-1}(s, u(s)) d s\right|\right]\right) \\
\leq & \lim _{n \rightarrow \infty}\left(\sup _{u(t) \in B}\left[\sum_{k \geq n} \int_{0}^{T}|G(t, s)|\left|k f_{k}(s, u(s))-(k-1) f_{k-1}(s, u(s))\right| d s\right]\right) \\
\leq & \lim _{n \rightarrow \infty}\left(\sup _{u(t) \in B}\left[\sum_{k \geq n} \int_{0}^{T}|G(t, s)|\left(g_{k}(s)+h_{k}(s)\left|k u_{k}(s)-(k-1) u_{k-1}(s)\right|\right]\right)\right. \\
= & \lim _{n \rightarrow \infty}\left(\sup _{u(t) \in B}\left[\sum_{k \geq n} \int_{0}^{T}|G(t, s)| g_{k}(s) d s+\sum_{k \geq n} \int_{0}^{T}|G(t, s)| h_{k}(s)\left|k u_{k}(s)-(k-1) u_{k-1}(s)\right| d s\right]\right) \\
\leq & \lim _{n \rightarrow \infty}\left(\sup _{u(t) \in B}\left[\int_{0}^{T}|G(t, s)| \sum_{k \geq n} g_{k}(s) d s+H \sum_{k \geq n}\left|k u_{k}-(k-1) u_{k-1}\right| \int_{0}^{T}|G(t, s)| d s\right]\right) \\
< & \lim _{n \rightarrow \infty} \frac{T}{\sin T} \sum_{k \geq n} g_{k}(s) d s+\frac{H T}{\sin T} \lim _{n \rightarrow \infty}\left(\sup _{u(t) \in B}\left[\sum_{k \geq n}\left|k u_{k}-(k-1) u_{k-1}\right|\right]\right) \\
= & \frac{H T}{\sin T} \mathcal{X}(\bar{B})
\end{aligned}
$$


Therefore,

$$
\mathcal{X}(\mathcal{F} \bar{B})<\mathcal{X}(\bar{B}) \frac{H T}{\sin T}<\epsilon \Longrightarrow \mathcal{X}(\bar{B})<\frac{\epsilon \sin T}{H T}
$$

Taking $\delta=\frac{\sin T-H T}{H T} \epsilon$, we get $\epsilon \leq \mathcal{X}(\bar{B})<\epsilon+\delta$. This shows that, $\mathcal{F}$ is a Meir-Keeler condensing operator defined on the set $\bar{B} \subset \int b v$ and so it satisfies all conditions of Theorem (2.3). Hence, $\mathcal{F}$ has fixed point in $\bar{B}$, which is a solution of the system (3.4).

Example 5.1. Let us consider the system of differential equations

$$
\frac{d^{2} u_{n}(t)}{d t^{2}}+u_{n}(t)=\frac{t}{n 3^{n} n !}+\sum_{m=n}^{\infty} \frac{\sqrt{t} \sin ^{2} t\left(m u_{m}(t)-(m-1) u_{m-1}(t)\right)}{(m+1)(m+2)(m+3)},
$$

$n \in \mathbf{N}, t \in[0, T], 0<T<\pi$ and $u_{i}(0)=u_{i}(T)=0$, for each $i$.

Clearly, the functions $\frac{t}{n 3^{n} n !}$ and $\frac{\sqrt{t} \sin ^{2} t\left(m u_{m}(t)-(m-1) u_{m-1}(t)\right)}{(m+1)(m+2)(m+3)}$ are continuous on $[0, T]$, for each $n \in \mathbf{N}$.

Here, $f_{n}(t, u(t))=\frac{t}{n 3^{n} n !}+\sum_{m=n}^{\infty} \frac{\sqrt{t} \sin ^{2} t\left(m u_{m}(t)-(m-1) u_{m-1}(t)\right)}{(m+1)(m+2)(m+3)}$.

Now,

$$
\begin{aligned}
& \sum_{k=1}^{\infty}\left|k f_{k}(t, u(t))-(k-1) f_{k-1}(t, u(t))\right| \\
= & \sum_{k=1}^{\infty} \mid \frac{t}{3^{k} k !}-\frac{t}{3^{k-1}(k-1) !} \\
& +k \sum_{m=k}^{\infty} \frac{\sqrt{t} \sin ^{2} t\left(m u_{m}(t)-(m-1) u_{m-1}(t)\right)}{(m+1)(m+2)(m+3)} \\
& -(k-1) \sum_{m=k-1}^{\infty} \frac{\sqrt{t} \sin ^{2} t\left(m u_{m}(t)-(m-1) u_{m-1}(t)\right)}{(m+1)(m+2)(m+3)} \mid \\
\leq & T \sum_{k=1}^{\infty}\left|\frac{1}{3^{k-1}(k-1) !} \frac{1-3 k}{3 k}\right|+T \sum_{k=1}^{\infty} \mid(1-k) \frac{\left(k u_{k}(t)-(k-1) u_{k-1}(t)\right)}{(k+1)(k+2)(k+3)} \\
& +\sum_{m=k}^{\infty} \frac{\left(m u_{m}(t)-(m-1) u_{m-1}(t)\right)}{(m+1)(m+2)(m+3)} \mid \\
\leq & T e^{\frac{1}{3}}+2 T \sum_{k=1}^{\infty} \frac{k\left|k u_{k}(t)-(k-1) u_{k-1}\right|}{(k+1)(k+2)(k+3)} \\
\leq & T e^{\frac{1}{3}}+2 T|| u(t)|| \int b v<\infty
\end{aligned}
$$


Therefore, for any $t \in[0, T],\left(f_{i}(t, u(t))\right) \in \int b v$.

We will show that the assumption (H3) is satisfied.

Let $\epsilon>0$ be given and $u(t)=\left(u_{n}(t)\right) \in \int b v$. Then taking $v(t)=\left(v_{n}(t)\right) \in$ $\int b v$ with $\|u(t)-v(t)\|_{\int b v} \leq \delta(\epsilon)=\frac{\epsilon}{T}$, we have

$$
\begin{aligned}
\mid f(t, u(t)) & -f(t, v(t)) \mid \\
& =\sum_{m=n}^{\infty} \frac{\sqrt{t}\left(\left(m u_{m}(t)-(m-1) u_{m-1}(t)\right)-\left(m v_{m}(t)-(m-1) v_{m-1}(t)\right)\right)}{(m+1)(m+2)(m+3)} \\
& \leq \sum_{m=1}^{\infty} \frac{\sqrt{t}\left(m\left(u_{m}(t)-v_{m}(t)\right)-(m-1)\left(u_{m-1}(t)-v_{m-1}(t)\right)\right)}{(m+1)(m+2)(m+3)} \\
& \leq T\|u(t)-v(t)\|_{\int b v} \\
& \leq T \delta<\epsilon,
\end{aligned}
$$

which implies continuity as assumed in (H3).

Now, we show that assumption (H4) is satisfied:

$$
\begin{aligned}
& \left|n f_{n}(t, u(t))-(n-1) f_{n-1}(t, u(t))\right| \\
= & \mid \frac{t}{3^{n} n !}-\frac{t}{3^{n-1}(n-1) !}+n \sum_{m=n}^{\infty} \frac{\sqrt{t} \sin ^{2} t\left(m u_{m}(t)-(m-1) u_{m-1}(t)\right)}{(m+1)(m+2)(m+3)} \\
& -(n-1) \sum_{m=n-1}^{\infty} \frac{\sqrt{t} \sin ^{2} t\left(m u_{m}(t)-(m-1) u_{m-1}(t)\right)}{(m+1)(m+2)(m+3)} \mid \\
\leq & \left|\frac{t}{3^{n-1}(n-1) !} \frac{1-3 n}{3 n}\right|+n \sum_{m=1}^{\infty}\left|\frac{\sqrt{t}\left(m u_{m}(t)-(m-1) u_{m-1}(t)\right)}{(m+1)(m+2)(m+3)}\right| \\
& +(n-1) \sum_{m=1}^{\infty}\left|\frac{\sqrt{t}\left(m u_{m}(t)-(m-1) u_{m-1}(t)\right)}{(m+1)(m+2)(m+3)}\right| \\
\leq & \frac{t}{3^{n-1}(n-1) !}+(2 n-1) \sum_{m=1}^{\infty} \frac{\sqrt{t}\left|\left(m u_{m}(t)-(m-1) u_{m-1}(t)\right)\right|}{(m+1)(m+2)(m+3)} \\
\leq & g_{n}(t)+h_{n}(t)\left|n u_{n}(t)-(n-1) u_{n-1}(t)\right| .
\end{aligned}
$$

The function $g_{n}(t)=\frac{t}{3^{n-1}(n-1) !}$ is continuous and $\sum_{n \geq 1} g_{n}(t)$ converges uniformly to $t e^{1 / 3}$. Also, $h_{n}(t)=\frac{\sqrt{t}}{12}$ is continuous and the sequence $\left(h_{n}(t)\right)$ is equibounded on $I$ by $H=\frac{\sqrt{T}}{12}$. Also $\frac{H T}{\sin T}<1$ is satisfied by taking $T=2.73$ (i.e. $0 \leq T \leq 0.7<\pi$ ), which gives $H \approx 0.12693$ and $G \approx 3.81002$. 
Thus, from the Theorem 5.1, for some suitable value of $r$, the operator $\mathcal{F}$ as defined in Theorem 5.1 on $\bar{B}\left(u^{0}, r\right)$ has a fixed point $u(t)=\left(u_{i}(t)\right) \in \int b v$, which is a solution of system of equations (5.1).

\section{References}

[1] K. Kuratowski, "Sur les espaces complets", Fundamenta mathematicae, vol. 15, no. 1, pp. 301-309, 1930. [On line]. Available: https:// bit.ly/ 3cxtQr5

[2] G. Darbo, "Punti uniti in transformazioni a condominio non compatto", Rendiconti del Seminario Matematico della Università di Padova, vol. 24, pp. 84-92, 1955. [On line]. Available: https:/ / bit.ly/ 3ctf8kM

[3] L. S. Goldenstein and A. S. Markus, "On a measure of noncompactness of bounded sets and linear operators", Studies in algebra and mathematical analysis, vol. 29, pp. 45-54, 1965.

[4] A. Meir and E. Keeler, "A theorem on contraction mappings", Journal of mathematics analysis and applications, vol. 28, no. 1, 1969, pp. 326329, 1969, doi: 10.1016/ 0022-247X(69)90031-6

[5] G. Goes and S. Goes, "Sequences of bounded variation and sequences of Fourier coefficients. I", Mathematische zeitschrift, vol. 118, no. 2, pp. 93-102, 1970, doi: 10.1007/ BF01110177

[6] V. Istrățescu, "On a measure of noncompactness", Bulletin mathématique de la Société des Sciences Mathématiques de la République Socialiste de Roumanie, Nouvelle Série, vol. 16, no. 2, pp. 195-197, 1972. [On line]. Available: https:/ / bit.ly/ 3vlMcDQ

[7] J. Banaś and K. Goebel, Measures of noncompactness in Banach spaces. New York, NY: Marcel Dekker, 1980.

[8] K. P. Persidskii, "Countable systems of differential equations and stability of their solutions", Izvestiya Akademii Nauk Kazakh SSR, vol. 7, pp. 52-71, 1959.

[9] K. P. Persidskii, "Countable systems of differential equations and stability of their solutions III: fundamental theorems on stability of countable many differential equations", Izvestiya Akademii Nauk Kazakh SSR, vol. 9, pp. 11-34, 1961.

[10] K. P. Persidskii, "Infinite system of differential equations", Izvestiya Akademii Nauk Kazakh SSR Alma Ata Differential Equations. Nonlinear Spaces, 1976. 
[11] J. Banaś, "Measures of noncompactness in the space of continuous tempered functions", Demonstratio mathematica, vol. 14, no. 1, pp. 127-133, 1981, doi: 10.1515/ dema-1981-0110

[12] R. R. Akhmerov, M. I. Kamenskii, A. S. Potapov, A. E. Rodkina, and B. N. Sadovskii, Measures of noncompactness and condensing operators, operator theory: advances and applications. Basel: Birkhäauser, 1992, doi: 10.1007/978-3-0348-5727-7

[13] J. Banaś and A. Martinon, "Some properties of the Hausdorff distance in metric spaces", Bulletin of the Australian Mathematical Society, vol. 42, no. 3, pp. 511-516, 1990, doi: 10.1017/ S0004972700028677

[14] J. Banaś and A. Martinon, "Measures of noncompactness in Banach sequence spaces", Mathematica Slovaca, vol. 42, no. 4, pp. 497-493, 1992. [On line]. Available: https:/ / bit.ly/ 3qZ6PTb

[15] J. Banaś and M.Lecko, "Solvability of infinite systems of differential equations in Banach sequence spaces", Journal of computational and applied mathematics, vol. 137, no. 2, pp. 363-375, 2001, doi: 10.1016/ S0377-0427(00)00708-1

[16] D. G. Duffy, Greens functions with applications. Boca Raton, FL: Chapman \& Hall/ CRC, 2001.

[17] R. P. Agarwal, M. Benchohra, and D. Seba, "On the application of measure of noncompactness to the existence of solutions for fractional differential equations", Results in mathematics, vol. 55, no. 3-4, pp. 221-230, 2009, doi: 10.1007/ s00025-009-0434-5

[18] M. Mursaleen and A. K. Noman, "Compactness by the Hausdorff measure of noncompactness", Nonlinear analysis: theory, methods \& applications, vol. 73, no. 8, pp. 2541-2557, 2010, doi: 10.1016/j.na.2010.06. 030

[19] M. Mursaleen and S. A. Mohiuddine, "Applications of measures of noncom-pactness to the infinite system of differential equations in $\ell_{p}$ spaces", Nonlinear analysis: theory, methods \& applications, vol. 75, no. 4, pp. 2111-2115, 2012, doi: 10.1016/ j.na.2011.10.011

[20] J. Banaś, and M. Mursaleen, Sequence spaces and measures of noncompactness with applications to differential and integral equations. New Delhi: Springer, 2014, doi: 10.1007/978-81-322-1886-9 
[21] A. Aghajani, R. Allahyari, and M. Mursaleen, "A generalization of Darbos theorem with application to the solvability of systems of integral equations", Journal of computational and applied mathematics, vol. 260, pp. 68-77, 2014, doi: 10.1016/j.cam.2013.09.039

[22] A. Aghajani, M. Mursaleen, and A. S. Haghighi, "Fixed point theorems for Meir-Keeler condensing operators via measure of noncompactness", Acta mathematica scientia, vol. 35, no. 3, pp. 552-566, 2015, doi: 10.1016/ S0252-9602(15)30003-5

[23] A. Alotaibi, M. Mursaleen, and S. A. Mohiuddine, "Application of measure of noncompactness to infinite system of linear equations in sequence spaces", Bulletin of the Iranian Mathematical Society, vol. 41, no. 2, pp. 519-527, 2015. [On line]. Available: https:/ / bit.ly/ 30MVB9F

[24] M. Kirişci, "Integrated and differentiated sequence spaces", Journal of nonlinear analysis and application, vol. 2015, no. 1, pp. 2-16, 2015, doi: 10.5899/2015/jnaa-00266

[25] M. Mursaleen and S. M. H. Rizvi, "Solvability of infinite systems of second order differential equations in $c_{0}$ and $\ell_{1}$ by Meir-Keeler condensing operators", Proceeding of the American Mathematical Society, vol. 144 , no. 10 , pp. 4279-4289, 2016, doi: $10.1090 / \mathrm{proc} /$ 13048

[26] H. M. Srivastava, A. Das, B. Hazarika, and S. A. Mohiuddine, "Existence of solutions of infinite systems of differential equations of general order with boundary conditions in the spaces $c_{0}$ and $\ell_{1}$ via the measure of noncompactness", Mathematical methods in the applied sciences, vol. 41, no. 10, pp. 3557-3569, 2018, doi: 10.1002/ mma.4845

[27] A. Das, B. Hazarika, and M. Mursaleen, "Application of measure of noncom-pactness for solvability of the infinite system of integral equations in two variables in $\ell_{p}(1<\mathrm{p}<\infty)$ ", Revista de la Real Academia de Ciencias Exactas, Físicas y Naturales. Serie A. Matemáticas, vol. 113, no. 1, pp. 31-40, 2019, doi: 10.1007/s13398-017-0452-1

[28] R. Saadati, E. Pourhadi, and M. Mursaleen, "Solvability of infinite systems of third-order differential equations in co by Meir-Keeler condensing operators", Journal of fixed point theory and applications, vol. 21, Art. ID. 64, 2019, doi: 10.1007/ s11784-019-0696-9

[29] B. Hazarika, R. Arab, and M. Mursaleen, "Application measure of noncompactness and operator type contraction for solvability of an infinite system of differential equations in $\ell_{p}$-space”, Filomat, vol. 33, no. 7, pp. 2181-2189, 2019, doi: 10.2298/ FIL1907181H 